\title{
SADDLEPOINT APPROXIMATION TO THE DISTRIBUTION OF THE TOTAL DISTANCE OF THE VON MISES-FISHER CONTINUOUS TIME RANDOM WALK
}

\author{
R. Gatto \\ Submission: July 2017 \\ Revisions: October 2017 and December 2017
}

\begin{abstract}
This article considers the random walk over $\mathbb{R}^{p}$, with any $p \geq 2$, where a particle starts at the origin and progresses stepwise with fixed step lengths and von Mises-Fisher distributed step directions. The total number of steps follows a continuous time counting process. The saddlepoint approximation to the distribution of the distance between the origin and the position of the particle at any time is derived. Despite the $p$-dimensionality of the random walk, the computation of the proposed saddlepoint approximation is one-dimensional and thus simple. The high accuracy of the saddlepoint approximation is illustrated by a numerical comparison with Monte Carlo simulation.
\end{abstract}

\section{Key words and phrases}

Bessel function; directional distribution; Legendre-Fenchel transform; Poisson process.

\section{Acknowledgment}

The author is grateful to two anonymous Referees for their important comments and suggestions which improved the quality of this article and to the Editor-in-Chief T. E. Simos.

MSC 2010

41A60 Asymptotic approximations, asymptotic expansions (steepest descent, etc.)

60G50 Sums of independent random variables; random walks

\section{PACS 2010}

02.30.Mv Approximations and expansions

05.40.Fb Random walks and Lévy flights

\section{Addresses}

Institute of Mathematical Statistics and Actuarial Science

Department of Mathematics and Statistics

University of Bern

Alpeneggstrasse 22, 3012 Bern, Switzerland

$+41316318807$

gatto@stat.unibe.ch www.stat.unibe.ch/gatto orcid.org/0000-0001-8374-6964 


\section{Introduction}

A particle in $\mathbb{R}^{p}$, with $p \geq 2$, starts at the origin and proceeds stepwise with fixed step lengths and along step directions that follow a prescribed directional distribution. This is the random flight or random walk, which appears in some problems of statistical mechanics and crystallography, refer e.g. to Shmueli and Weiss (1995). The determination of the distribution of the total distance between the origin and the position of the particle after a given number of steps is a classical problem, dating from the beginning of the 20-th century. When the number steps arises from an independent continuous time counting process, this random walk is a particular continuous time random walk (c.t.r.w). The c.t.r.w. was introduced by Montroll and Weiss (1965) as a model for anomalous diffusion. The present article provides a saddlepoint approximation to the distribution of the distance to the origin of the c.t.r.w. with arbitrary dimension $p \geq 2$ and when the directions of the steps of the particle follow the von Mises-Fisher directional distribution.

The proposed saddlepoint approximation relies on the saddlepoint approximation to the density of the sample mean introduced by Daniels (1954). This saddlepoint approximation is very accurate, substantially more than the asymptotic normal approximation and in particular for approximating small tail probabilities. This is due to the fact that the relative error is bounded. Two general references are Jensen (1995) and Sections 4.3 and 6.5 of Barndorff-Nielsen and Cox (1989). Therefore the saddlepoint approximation provides a computational efficient alternative to purely numerical methods, like Monte Carlo simulation. The von Mises-Fisher distribution is the central model for the analysis of directional data. It shares many theoretical properties or characterizations with the Gaussian distribution of the Euclidean space. It is fully presented in e.g. Section 9.3.2 of Mardia and Jupp (2000).

The saddlepoint approximation of this article generalizes the one for the planar c.t.r.w. of Gatto (2017a) from $p=2$ to any dimension $p \geq 2$. Because any planar direction can be represented by an angle, in radians for example, any directional distribution on the plane is usually represented by a distribution over $[0,2 \pi)$. However with any higher dimension $p \geq 3$, the unit vector representation directions appears more convenient than the angular one and the results of Gatto (2017a) cannot be directly generalized. The proposed saddlepoint approximation generalizes also the multidimensional one with fixed number of steps of Gatto (2017b). Gatto (2017c) proposes a saddlepoint approximation for the c.t.r.w. with arbitrary dimension $p \geq 2$, with random step lengths but with isotropic viz. uniform step directions. The isotropic distribution is a special case of the von Mises-Fisher and so this article provides a generalization in this sense. Recent surveys of the literature on alternative or related approximations can be found e.g. in Gatto (2017a, 2017b, 2017c).

The derivation of the new saddlepoint approximation is given in Section 2. Some standard formulae and results related to directional distributions are given in Section 2.1. The saddlepoint approximation of the c.t.r.w. with von Mises-Fisher directions is given 
in Section 2.2. Section 3 shows some numerical comparisons of the proposed saddlepoint approximation with the distribution obtained by simulation.

\section{The saddlepoint approximation}

Some central formulae of directional distributions are given in Section 2.1. The von MisesFisher distribution is introduced. The formulae of Section 2.1 are used in Section 2.2, which provides the derivation of the saddlepoint approximation to the distribution of the total distance of the c.t.r.w. with von Mises-Fisher directions.

\subsection{Directional distributions}

For $p \geq 3, \boldsymbol{y} \in \mathbb{R}^{p} \backslash\{0\}$ can be re-expressed as $\boldsymbol{y}=\boldsymbol{g}\left(\alpha_{1}, \ldots, \alpha_{p-1}, r\right)$, where

$$
\begin{aligned}
y_{1}= & r \sin \alpha_{1} \sin \alpha_{2} \ldots \sin \alpha_{p-2} \cos \alpha_{p-1}, \\
y_{2}= & r \sin \alpha_{1} \sin \alpha_{2} \ldots \sin \alpha_{p-2} \sin \alpha_{p-1}, \\
\vdots & \vdots \\
y_{p-1}= & r \sin \alpha_{1} \sin \alpha_{2}, \\
y_{p}= & r \cos \alpha_{1},
\end{aligned}
$$

where $0 \leq \alpha_{j} \leq \pi$, for $j=1, \ldots, p-2,0 \leq \alpha_{p-1}<2 \pi$ and $r>0$. For $p=2, \boldsymbol{y} \in \mathbb{R}^{2} \backslash\{0\}$ can be re-expressed as $\boldsymbol{y}=\boldsymbol{g}\left(\alpha_{1}, r\right)$, where $y_{1}=r \sin \alpha_{1}, y_{2}=r \cos \alpha_{1}$, where $0 \leq \alpha_{1}<2 \pi$ and $r>0$. The determinant of the Jacobian matrix of $\boldsymbol{g}$ is given by

$$
J\left(\alpha_{1}, \ldots, \alpha_{p-2}, r\right)=r^{p-1} \prod_{j=2}^{p-1} \sin ^{p-j} \alpha_{j-1} .
$$

In this formula and later in this article, we use the convention that the argument $\alpha_{p-2}$ and the product $\prod_{j=2}^{p-1}$ must be ignored when $p=2$. Define the centered sphere of radius $r>0$ by $\mathbb{S}_{r}^{p-1}=\left\{\boldsymbol{y} \in \mathbb{R}^{p} \mid\|\boldsymbol{y}\|=r\right\}$. For any continuous function $z: \mathbb{S}_{r}^{p-1} \rightarrow \mathbb{R}$,

$$
\int_{\mathbb{S}_{r}^{p-1}} z(\boldsymbol{y}) \mathrm{d} \lambda_{p, r}(\boldsymbol{y})=\int_{0}^{2 \pi} \int_{0}^{\pi} \ldots \int_{0}^{\pi} z\left(\boldsymbol{g}\left(\alpha_{1}, \ldots, \alpha_{p-1}, r\right)\right) J\left(\alpha_{1}, \ldots, \alpha_{p-2}, r\right) \mathrm{d} \alpha_{1} \ldots \mathrm{d} \alpha_{p-1},
$$

where $\mathrm{d} \lambda_{p, r}(\boldsymbol{y})$ denotes the infinitesimal surface area, or Lebesgue measure, around $\boldsymbol{y} \in \mathbb{S}_{r}^{p-1}$ and over $\mathbb{S}_{r}^{p-1}$. In this formula and later in this article, we use the convention that the above integrals from 0 to $\pi$ must be ignored when $p=2$. Thus the surface area of $\mathbb{S}_{r}^{p-1}$ is given by

$$
a_{p, r}=\int_{\mathbb{S}_{r}^{p-1}} \mathrm{~d} \lambda_{p, r}(\boldsymbol{y})=r^{p-1} \frac{2 \pi^{\frac{p}{2}}}{\Gamma\left(\frac{p}{2}\right)}
$$


Denote $\mathbb{S}^{p-1}=\mathbb{S}_{1}^{p-1}$ and $\mathcal{B}\left(\mathbb{S}^{p-1}\right)=\left\{B \cap \mathbb{S}^{p-1} \mid B \in \mathcal{B}\left(\mathbb{R}^{p}\right)\right\}$ the Borel $\sigma$-field. The uniform or isotropic distribution over $\left(\mathbb{S}^{p-1}, \mathcal{B}\left(\mathbb{S}^{p-1}\right)\right)$ is given by $U_{p}[B]=\int_{B} a_{p}^{-1} \mathrm{~d} \lambda_{p}(\boldsymbol{x})=$ $a_{p}^{-1} \lambda_{p}[B], \forall B \in \mathcal{B}\left(\mathbb{S}^{p-1}\right)$, where $a_{p}=a_{p, 1}$ and $\lambda_{p}=\lambda_{p, 1}$. The von Mises-Fisher distribution over $\left(\mathbb{S}^{p-1}, \mathcal{B}\left(\mathbb{S}^{p-1}\right)\right)$, with mean direction $\boldsymbol{\mu} \in \mathbb{S}^{p-1}$ and concentration $\kappa \geq 0$, has density with respect to (w.r.t.) the isotropic distribution $U_{p}$ given by

$$
f_{p}(\boldsymbol{x} \mid \boldsymbol{\mu}, \kappa)=c_{p}(\kappa) \exp \{\kappa\langle\boldsymbol{\mu}, \boldsymbol{x}\rangle\}, \quad \forall \boldsymbol{x} \in \mathbb{S}^{p-1},
$$

where

$$
c_{p}(u)= \begin{cases}\left(\frac{|u|}{2}\right)^{\frac{p}{2}-1}\left\{\Gamma\left(\frac{p}{2}\right) I_{\frac{p}{2}-1}(|u|)\right\}^{-1}, & \text { if } u \in \mathbb{R} \backslash\{0\}, \\ 1, & \text { if } u=0 .\end{cases}
$$

Recall that $I_{\nu}(z)$ is the modified Bessel function $I$ of order $\nu, \forall z \in \mathbb{C}$ and $\nu \in \mathbb{C}$ such that $\Re \nu>-1 / 2$; see e.g. Abramowitz and Stegum (1972), 9.6.18, p. 376. The density w.r.t. $\lambda_{p}$ has normalizing constant $a_{p}^{-1} c_{p}(\kappa)$. We use the abbreviation $\operatorname{vMF}(\boldsymbol{\mu}, \kappa)$ distribution for the von Mises-Fisher distribution with mean direction $\boldsymbol{\mu}$ and concentration $\kappa$.

\subsection{Derivation of the saddlepoint approximation}

We denote by $\boldsymbol{X}_{1}, \boldsymbol{X}_{2}, \ldots$ independent directional random vectors taking values in $\mathbb{S}^{p-1}$ and with a common absolutely continuous distribution w.r.t. $U_{p}$. The resultant vector of the $n$ first directions is $\boldsymbol{R}_{n}=\sum_{j=1}^{n} \boldsymbol{X}_{j}$, its length $R_{n}=\left\|\boldsymbol{R}_{n}\right\|$ and its direction $\boldsymbol{M}_{n}=\boldsymbol{R}_{n} / R_{n}$. We denote by $\left\{N_{t}\right\}_{t \geq 0}$ a continuous time counting process, i.e. an a.s. nondecreasing continuous time process taking nonnegative integer values. It is reasonable to assume $N_{0}=0$ a.s., so that only the times $t>0$ are interesting. We assume that the sigma fields $\sigma\left(\cup_{t \geq 0} \sigma\left(N_{t}\right)\right)$ and $\sigma\left(\cup_{j=1}^{\infty} \sigma\left(\boldsymbol{X}_{j}\right)\right)$ are independent which means that the counting process is independent of the elementary directions. We define $\boldsymbol{X}_{0}=\mathbf{0}$ and denote $\hat{\boldsymbol{R}}_{t}=\sum_{j=0}^{N_{t}} \boldsymbol{X}_{j}$, $\hat{R}_{t}=\left\|\hat{\boldsymbol{R}}_{t}\right\|$, viz. the total distance traveled by the particle from the time 0 to the time $t>0$, and $\hat{\boldsymbol{M}}_{t}=\hat{\boldsymbol{R}}_{t} / \hat{R}_{t}$, when $\hat{R}_{t}>0$. The Lebesgue measure over $\mathbb{R}^{p}$ is denoted by $l_{p}$.

The following basic result can be found for example in Gatto (2017c).

Lemma 2.1 (First polar factorization lemma). Let $n \geq 2$ and $\boldsymbol{X}_{1}, \ldots, \boldsymbol{X}_{n}$ be independent isotropic directional random vectors. Let $w_{p, n}$ be the joint density of $\left(\boldsymbol{M}_{n}, R_{n}\right)$ w.r.t. $U_{p} \times l_{1}$ and let $q_{p, n}$ be the density of $R_{n}$, w.r.t. $l_{1}$. Then

$$
w_{p, n}(\boldsymbol{x}, r)=q_{p, n}(r), \forall \boldsymbol{x} \in \mathbb{S}^{p-1}, r \in(0, n] .
$$

Assign the $\operatorname{vMF}(\boldsymbol{\mu}, \kappa)$ distribution with density (5) to the elementary directions $\boldsymbol{X}_{1}, \boldsymbol{X}_{2}$, .... Denote by $q_{\kappa, p, n}$ the Lebesgue density of the resultant length $R_{n}$, under the $\operatorname{vMF}(\boldsymbol{\mu}, \kappa)$ distribution. Then $q_{0, p, n}=q_{p, n}$. The joint density of $\left(\boldsymbol{M}_{n}, R_{n}\right)$ w.r.t. $U_{p} \times l_{1}$ and at 
$(\boldsymbol{x}, r) \in \mathbb{S}^{p-1} \times(0, n]$ is denoted $w_{\boldsymbol{\mu}, \kappa, p, n}(\boldsymbol{x}, r)$. It can be obtained as follows,

$$
\begin{aligned}
w_{\boldsymbol{\mu}, \kappa, p, n}(\boldsymbol{x}, r) & =\int \ldots \int_{\boldsymbol{x}_{1}+\ldots+\boldsymbol{x}_{n}=r \boldsymbol{x}} c_{p}^{n}(\kappa) \exp \left\{\kappa\left\langle\boldsymbol{\mu}, \boldsymbol{x}_{1}+\ldots+\boldsymbol{x}_{n}\right\rangle\right\} \mathrm{d} U_{p}\left(\boldsymbol{x}_{1}\right) \ldots \mathrm{d} U_{p}\left(\boldsymbol{x}_{n}\right) \\
& =c_{p}^{n}(\kappa) \exp \{\kappa r\langle\boldsymbol{\mu}, \boldsymbol{x}\rangle\} w_{\boldsymbol{\mu}, 0, p, n}(\boldsymbol{x}, r) \\
& =c_{p}^{n}(\kappa) \exp \{\kappa r\langle\boldsymbol{\mu}, \boldsymbol{x}\rangle\} q_{0, p, n}(r)
\end{aligned}
$$

where the last equality follows from the First polar factorization lemma 2.1. Because the density of $R_{n}$ at $r \in(0, n]$ is given by $q_{\kappa, p, n}(r)=\int_{\mathbb{S} p-1} w_{\boldsymbol{\mu}, \kappa, p, n}(\boldsymbol{x}, r) \mathrm{d} U_{p}(\boldsymbol{x}),(7)$ leads to the following result.

Lemma 2.2 (Tilting lemma). The densities of $R_{n}$ w.r.t. $l_{1}$, under isotropy and under the $\operatorname{vMF}(\boldsymbol{\mu}, \kappa)$ distribution, respectively denoted $q_{p, n}$ and $q_{\kappa, p, n}$, satisfy the relation

$$
q_{\kappa, p, n}(r)=\frac{c_{p}^{n}(\kappa)}{c_{p}(\kappa r)} \cdot q_{p, n}(r), \quad \forall r \in(0, n]
$$

Define $p_{n}(t)=\mathrm{P}\left[N_{t}=n\right], \forall n \in\{0,1, \ldots\}$ and $t \geq 0$. Let $t>0$ and denote by $N_{t}^{0}$ the zero-truncation of $N_{t}$ : set $N_{t}^{0}=N_{t}$ over $\left\{N_{t}>0\right\}$ and renormalize its distribution. Thus the distribution of $N_{t}^{0}$ is given by $p_{n}^{0}(t)=\mathrm{P}\left[N_{t}^{0}=n\right]=p_{n}(t) /\left\{1-p_{0}(t)\right\}, \forall n \in\{1,2, \ldots\}$ and $t>0$. Denote by $\hat{q}_{\kappa, p, t}$ the conditional density of $\hat{R}_{t}$ given $\left\{N_{t}>0\right\}$, w.r.t. $l_{1}$ and under the $\operatorname{vMF}(\boldsymbol{\mu}, \kappa)$ distribution. Denote by $\hat{g}_{\boldsymbol{\mu}, \kappa, p, t}$ the conditional density of $\hat{\boldsymbol{R}}_{t}$ given $\left\{N_{t}>0\right\}$, w.r.t. $l_{p}$ and under the $\operatorname{vMF}(\boldsymbol{\mu}, \kappa)$ distribution.

Lemma 2.3 (Second polar factorization lemma). Let $t>0$. Under the $\operatorname{vMF}(\boldsymbol{\mu}, \kappa)$ distribution, with density given by (5), the conditional joint density of $\left(\hat{\boldsymbol{M}}_{t}, \hat{R}_{t}\right)$ given $\left\{N_{t}>0\right\}$ and w.r.t. $U_{p} \times l_{1}$ factorizes as

$$
\hat{w}_{\boldsymbol{\mu}, \kappa, p, t}(\boldsymbol{x}, r)=f_{p}(\boldsymbol{x} \mid \boldsymbol{\mu}, \kappa r) \cdot \hat{q}_{\kappa, p, t}(r), \forall \boldsymbol{x} \in \mathbb{S}^{p-1} \text { and } r>0 .
$$

Alternatively, consider the angular polar coordinates of $\hat{\boldsymbol{R}}_{t}$ given in (1). Let $\hat{w}_{\boldsymbol{\mu}, \kappa, p, t}^{\circ}$ be the conditional joint Lebesgue density of the polar angles $\left(\hat{\alpha}_{1, t}, \ldots, \hat{\alpha}_{p-1, t}\right)$ and of the resultant length $\hat{R}_{t}$ of $\hat{\boldsymbol{R}}_{t}$, given $\left\{N_{t}>0\right\}$. Then this density factorizes as

$$
\hat{w}_{\boldsymbol{\mu}, \kappa, p, t}^{\circ}\left(\alpha_{1}, \ldots, \alpha_{p-1}, r\right)=a_{p}^{-1} f_{p}\left(\boldsymbol{g}\left(\alpha_{1}, \ldots, \alpha_{p-1}, 1\right) \mid \boldsymbol{\mu}, \kappa r\right) \prod_{j=2}^{p-1} \sin ^{p-j} \alpha_{j-1} \cdot \hat{q}_{\kappa, p, t}(r),
$$

$\forall \alpha_{1}, \ldots, \alpha_{p-2} \in[0, \pi], \alpha_{p-1} \in[0,2 \pi)$ and $r>0$, where $\boldsymbol{g}$ is the diffeomorphism (1) and where $a_{p}=a_{p, 1}$ is given by (4).

We denote by $P_{\boldsymbol{\mu}, \kappa}$ the probability measure under the $\operatorname{vMF}(\boldsymbol{\mu}, \kappa)$ distribution.

Proof. Let $r, t>0$ and $\boldsymbol{x} \in \mathbb{S}^{p-1}$, then from (7), for the fourth equality in (10), and from 
the Tilting lemma 2.2, for the last equality in (10), it follows that

$$
\begin{aligned}
\hat{w}_{\boldsymbol{\mu}, \kappa, p, t}(\boldsymbol{x}, r) \mathrm{d} U_{p}(\boldsymbol{x}) \mathrm{d} & l_{1}(r)=\mathrm{P}_{\boldsymbol{\mu}, \kappa}\left[\hat{\boldsymbol{M}}_{t} \in B^{p-1}\left(\boldsymbol{x}, \mathrm{d} \lambda_{p}(\boldsymbol{x})\right), \hat{R}_{t} \in\left(r, r+\mathrm{d} l_{1}(r)\right) \mid N_{t}>0\right] \\
& =\frac{\sum_{n=0}^{\infty} \mathrm{P}_{\boldsymbol{\mu}, \kappa}\left[\hat{\boldsymbol{M}}_{t} \in B^{p-1}\left(\boldsymbol{x}, \mathrm{d} \lambda_{p}(\boldsymbol{x})\right), \hat{R}_{t} \in\left(r, r+\mathrm{d} l_{1}(r)\right), N_{t}=n, N_{t}>0\right]}{\mathrm{P}\left[N_{t}>0\right]} \\
& =\sum_{n=1}^{\infty} w_{\boldsymbol{\mu}, \kappa, p, n}(\boldsymbol{x}, r) \mathrm{d} U_{p}(\boldsymbol{x}) \mathrm{d} l_{1}(r) p_{n}^{0}(t) \\
& =\sum_{n=1}^{\infty} c_{p}^{n}(\kappa) \exp \{\kappa r\langle\boldsymbol{\mu}, \boldsymbol{x}\rangle\} q_{0, p, n}(r) \mathrm{d} U_{p}(\boldsymbol{x}) \mathrm{d} l_{1}(r) p_{n}^{0}(t) \\
& =f_{p}(\boldsymbol{x} \mid \boldsymbol{\mu}, \kappa r) \sum_{n=1}^{\infty} \frac{c_{p}^{n}(\kappa)}{c_{p}(\kappa r)} q_{0, p, n}(r) \mathrm{d} U_{p}(\boldsymbol{x}) \mathrm{d} l_{1}(r) p_{n}^{0}(t) \\
& =f_{p}(\boldsymbol{x} \mid \boldsymbol{\mu}, \kappa r) \mathrm{d} U_{p}(\boldsymbol{x}) \sum_{n=1}^{\infty} q_{\kappa, p, n}(r) p_{n}^{0}(t) \mathrm{d} l_{1}(r),
\end{aligned}
$$

where $B^{p-1}(\boldsymbol{x}, s)$ is an arbitrary open set of $\mathbb{S}^{p-1}$ containing $\boldsymbol{x}$ and possessing surface area $s>0$. Integrating the last expression over $\mathbb{S}^{p-1}$ yields

$$
\hat{q}_{\kappa, p, t}(r) \mathrm{d} l_{1}(r)=\sum_{n=1}^{\infty} q_{\kappa, p, n}(r) p_{n}^{0}(t) \mathrm{d} l_{1}(r) .
$$

Then (8) is obtained after inserting (11) into (10).

In order to see (9), use (3) to write, $\forall r>0$,

$$
\begin{aligned}
\int_{\mathbb{S}^{p-1}} & \hat{w}_{\boldsymbol{\mu}, \kappa, p, t}(\boldsymbol{x}, r) a_{p}^{-1} \mathrm{~d} \lambda_{p}(\boldsymbol{x})= \\
& \int_{0}^{2 \pi} \int_{0}^{\pi} \ldots \int_{0}^{\pi} \hat{w}_{\boldsymbol{\mu}, \kappa, p, t}\left(\boldsymbol{g}\left(\alpha_{1}, \ldots, \alpha_{p-1}, 1\right), r\right) a_{p}^{-1} J\left(\alpha_{1}, \ldots, \alpha_{p-2}, 1\right) \mathrm{d} \alpha_{1} \ldots \mathrm{d} \alpha_{p-1} .
\end{aligned}
$$

From the factorization (8), this is equal to

$$
\int_{0}^{2 \pi} \int_{0}^{\pi} \ldots \int_{0}^{\pi} a_{p}^{-1} f_{p}\left(\boldsymbol{g}\left(\alpha_{1}, \ldots, \alpha_{p-1}, 1\right) \mid \boldsymbol{\mu}, \kappa r\right) \hat{q}_{\kappa, p, t}(r) J\left(\alpha_{1}, \ldots, \alpha_{p-2}, 1\right) \mathrm{d} \alpha_{1} \ldots \mathrm{d} \alpha_{p-1} .
$$

But the integrand is $\hat{w}_{\boldsymbol{\mu}, \kappa, p, t}^{\circ}\left(\alpha_{1}, \ldots, \alpha_{p-1}, r\right)$.

We now define some useful functions. We first define

$$
A_{p}(u)=\frac{I_{\frac{p}{2}}(u)}{I_{\frac{p}{2}-1}(u)}, \quad \forall u \geq 0,
$$

which is a continuous increasing mapping from $[0, \infty)$ onto $[0,1)$. Define also by $M_{N_{t}^{0}}$ the moment generating function (m.g.f.) of $N_{t}^{0}$ and by $K_{N_{t}^{0}}=\log M_{N_{t}^{0}}$ its cumulant generating function (c.g.f.), $\forall t>0$. We define also the following functions:

$$
\bar{M}_{\kappa}(u)=\frac{c_{p}(\kappa)}{c_{p}(u)}, \bar{K}_{\kappa}(u)=\log \bar{M}_{\kappa}(u),
$$




$$
\begin{gathered}
\hat{\bar{K}}_{\kappa, t}(u)=K_{N_{t}^{0}} \circ \bar{K}_{\kappa}(u), \\
\hat{C}_{\kappa, p, t}(u)=\hat{\bar{K}}_{\kappa, t}^{\prime}(u),
\end{gathered}
$$

the Hessian matrix of $\hat{\bar{K}}_{\kappa, t}$ and its determinant by

$$
\hat{H}_{\kappa, p, t}(\boldsymbol{v})=\frac{\partial^{2}}{\partial \boldsymbol{v}^{\top} \partial \boldsymbol{v}} \hat{\bar{K}}_{\kappa, t}(u) \text { and } \hat{\sigma}_{\kappa, p, t}^{2}(u)=\operatorname{det} \hat{H}_{\kappa, p, t}(\boldsymbol{v})
$$

where $u=\|\boldsymbol{v}\|$ and $\boldsymbol{v} \in \mathbb{R}^{p}$.

Theorem 2.4 (Saddlepoint approximation to the density). Let $r$ and $t>0$, then the saddlepoint approximation to $\hat{q}_{\kappa, p, t}(r)$, the conditional density of $\hat{R}_{t}$ given $\left\{N_{t}>0\right\}$, w.r.t. $l_{1}$ and under the $\operatorname{vMF}(\boldsymbol{\mu}, \kappa)$ distribution, is given by

$$
\tilde{\hat{q}}_{\kappa, p, t}(r)=2^{1-\frac{p}{2}} \Gamma^{-1}\left(\frac{p}{2}\right) \hat{\sigma}_{\kappa, p, t}^{-1}(\bar{u}) M_{N_{t}^{0}}\left(\log \frac{c_{p}(\kappa)}{c_{p}(\bar{u})}\right) c_{p}^{-1}(\kappa r) r^{p-1} \mathrm{e}^{-\bar{u} r}
$$

where the saddlepoint $\bar{u}$ is the positive, continuous and increasing function of $r$ obtained by solving w.r.t. $u$ the equation

$$
\hat{C}_{\kappa, p, t}(u)=r
$$

where

$$
\begin{gathered}
\hat{C}_{\kappa, p, t}(u)=K_{N_{t}^{0}}^{\prime}\left(\log \frac{c_{p}(\kappa)}{c_{p}(u)}\right) A_{p}(u), \\
\hat{\sigma}_{\kappa, p, t}^{2}(u)=\hat{C}_{\kappa, p, t}^{\prime}(u)\left(\frac{\hat{C}_{\kappa, p, t}(u)}{u}\right)^{p-1}, \\
\hat{\sigma}_{\kappa, p, t}^{2}(\bar{u})=\left(\frac{r}{\bar{u}}\right)^{p-1}\left\{K_{N_{t}^{0}}^{\prime \prime}\left(\log \frac{c_{p}(\kappa)}{c_{p}(\bar{u})}\right) A_{p}^{2}(\bar{u})-r A_{p}(\bar{u})-\frac{r(p-1)}{\bar{u}}+\frac{r}{A_{p}(\bar{u})}\right\}
\end{gathered}
$$

and where the functions $c_{p}$ and $A_{p}$ are given by (6) and (12), respectively. Let $\hat{b}_{\kappa, p, t}^{-1}=$ $\int_{0}^{\infty} \tilde{\hat{q}}_{\kappa, p, t}(r) \mathrm{d} r$, then the normalized saddlepoint approximation is given by $\hat{b}_{\kappa, p, t} \tilde{\hat{q}}_{\kappa, p, t}(r)$.

We denote by $\mathrm{E}_{\boldsymbol{\mu}, \kappa}$ the expectation functional under the $\operatorname{vMF}(\boldsymbol{\mu}, \kappa)$ distribution. Proof. This proof is made of three main parts: I, II and III. Part I provides formulae for m.g.f., c.g.f. and for other important functions. Relationships between these functions are provided. Part II provides the $p$-dimensional saddlepoint approximation to the conditional density of the position of the particle. Part III extracts from this $p$-dimensional saddlepoint 
approximation the one-dimensional density of the total distance of the particle.

I. The m.g.f. of $\boldsymbol{X}_{1}$ at $\boldsymbol{v} \in \mathbb{R}^{p}$ is given by

$$
\begin{aligned}
M_{\boldsymbol{\mu}, \kappa}(\boldsymbol{v}) & =\mathrm{E}_{\boldsymbol{\mu}, \kappa}\left[\exp \left\{\left\langle\boldsymbol{v}, \boldsymbol{X}_{1}\right\rangle\right\}\right] \\
& =\int_{\mathbb{S}^{p-1}} \exp \{\langle\boldsymbol{v}, \boldsymbol{x}\rangle\} c_{p}(\kappa) \exp \{\kappa\langle\boldsymbol{\mu}, \boldsymbol{x}\rangle\} \mathrm{d} U_{p}(\boldsymbol{x}) \\
& =\frac{c_{p}(\kappa)}{c_{p}(\|\kappa \boldsymbol{\mu}+\boldsymbol{v}\|)} .
\end{aligned}
$$

Let $t>0$. The m.g.f. of $\hat{\boldsymbol{R}}_{t}$ conditional on $\left\{N_{t}>0\right\}$ at $\boldsymbol{v} \in \mathbb{R}^{p}$ is obtained as follows,

$$
\begin{aligned}
\hat{M}_{\boldsymbol{\mu}, \kappa, t}(\boldsymbol{v}) & =\mathrm{E}_{\boldsymbol{\mu}, \kappa}\left[\exp \left\{\left\langle\boldsymbol{v}, \hat{\boldsymbol{R}}_{t}\right\rangle\right\} \mid N_{t}>0\right] \\
& =\left(\mathrm{P}\left[N_{t}>0\right]\right)^{-1} \mathrm{E}_{\boldsymbol{\mu}, \kappa}\left[\mathrm{E}_{\boldsymbol{\mu}, \kappa}\left[\exp \left\{\left\langle\boldsymbol{v}, \hat{\boldsymbol{R}}_{t}\right\rangle\right\} \mathrm{I}\left\{N_{t}>0\right\} \mid N_{t}\right]\right] \\
& =\left(\mathrm{P}\left[N_{t}>0\right]\right)^{-1} \mathrm{E}_{\boldsymbol{\mu}, \kappa}\left[\mathrm{E}_{\boldsymbol{\mu}, \kappa}\left[\exp \left\{\left\langle\boldsymbol{v}, \hat{\boldsymbol{R}}_{t}\right\rangle\right\} \mid N_{t}\right] \mathrm{I}\left\{N_{t}>0\right\}\right] \\
& =\mathrm{E}_{\boldsymbol{\mu}, \kappa}\left[M_{\boldsymbol{\mu}, \kappa}^{N_{t}}(\boldsymbol{v}) \mid N_{t}>0\right] \\
& =\mathrm{E}_{\boldsymbol{\mu}, \kappa}\left[M_{\boldsymbol{\mu}, \kappa}^{N_{t}^{0}}(\boldsymbol{v})\right] \\
& =M_{N_{t}^{0}} \circ K_{\boldsymbol{\mu}, \kappa}(\boldsymbol{v}),
\end{aligned}
$$

where $M_{N_{t}^{0}}$ is the m.g.f. of $N_{t}^{0}, M_{\boldsymbol{\mu}, \kappa}$ is given by (21) and $K_{\boldsymbol{\mu}, \kappa}(\boldsymbol{v})=\log M_{\boldsymbol{\mu}, \kappa}(\boldsymbol{v})$ is the c.g.f. Recall that $K_{N_{t}^{0}}=\log M_{N_{t}^{0}}$. Thus (22) yields $\hat{K}_{\boldsymbol{\mu}, \kappa, t}(\boldsymbol{v})=K_{N_{t}^{0}} \circ K_{\boldsymbol{\mu}, \kappa}(\boldsymbol{v})$. It then follows from definition (13) and from (21) that $\bar{M}_{\kappa}(\|\kappa \boldsymbol{\mu}+\boldsymbol{v}\|)=M_{\boldsymbol{\mu}, \kappa}(\boldsymbol{v})$. Thus, $\bar{M}_{\kappa}(u)=M_{\boldsymbol{\mu}, \kappa}(\boldsymbol{v}-\kappa \boldsymbol{\mu})=c_{p}(\kappa) \int_{\mathbb{S}^{p-1}} \exp \{\langle\boldsymbol{v}, \boldsymbol{x}\rangle\} \mathrm{d} U_{p}(\boldsymbol{x})$, which is $c_{p}(\kappa)$ times the m.g.f. of the isotropic distribution. By using the recurrence relation

$$
u I_{\nu+1}(u)=u I_{\nu}^{\prime}(u)-\nu I_{\nu}(u)
$$

see e.g. Abramowitz and Stegum (1972), 9.6.26, p. 376, and by using (6), one obtains

$$
\frac{\mathrm{d}}{\mathrm{d} u} \frac{1}{c_{p}(u)}=\Gamma\left(\frac{p}{2}\right)\left(\frac{u}{2}\right)^{1-\frac{p}{2}} I_{\frac{p}{2}}(u)
$$

Thus it follows from (13) and (24) that

$$
\bar{K}_{\kappa}^{\prime}(u)=c_{p}(u) \frac{\mathrm{d}}{\mathrm{d} u} \frac{1}{c_{p}(u)}=c_{p}(u) \Gamma\left(\frac{p}{2}\right)\left(\frac{u}{2}\right)^{1-\frac{p}{2}} I_{\frac{p}{2}}(u)=A_{p}(u) .
$$

Define by $\overline{\boldsymbol{v}}$ the solution w.r.t. $\boldsymbol{v}$ of

$$
\frac{\partial}{\partial \boldsymbol{v}} \hat{\bar{K}}_{\kappa, t}(u)=\hat{C}_{\kappa, p, t}(u) \frac{\partial u}{\partial \boldsymbol{v}}=K_{N_{t}^{0}}^{\prime} \circ \bar{K}_{\kappa}(u) \bar{K}_{\kappa}^{\prime}(u) \boldsymbol{z}=\boldsymbol{y},
$$

where $u=\|\boldsymbol{v}\|$ and $\boldsymbol{z}=\boldsymbol{v} / u$, where $\hat{\bar{K}}_{\kappa, t}$ and $\hat{C}_{\kappa, p, t}$ are respectively defined by (14) and (15). Let $\bar{u}=\|\overline{\boldsymbol{v}}\|$ and $\overline{\boldsymbol{z}}=\overline{\boldsymbol{v}} / \bar{u}$. Let $r=\|\boldsymbol{y}\|$ and $\boldsymbol{x}=\boldsymbol{y} / r$. It follows from (25) and (26) that $\bar{u}$ is the solution w.r.t. $u$ of

$$
K_{N_{t}^{0}}^{\prime} \circ \bar{K}_{\kappa}(u) A_{p}(u)=r
$$


i.e. of (18), and $\overline{\boldsymbol{z}}=\boldsymbol{x}$. Denote by $I_{p \times p}$ the identity matrix of size $p \times p$ and assume that $\boldsymbol{v}$ is a $p \times 1$ matrix. Recalling the definitions (16), we find

$$
\begin{aligned}
\hat{\sigma}_{\kappa, p, t}^{2}(u) & =\operatorname{det}\left(\frac{\hat{C}_{\kappa, p, t}(u)}{u}\left[\left\{\frac{u \hat{C}_{\kappa, p, t}^{\prime}(u)}{\hat{C}_{\kappa, p, t}(u)}-1\right\} \frac{\boldsymbol{v} \boldsymbol{v}^{\top}}{u^{2}}+I_{p \times p}\right]\right) \\
& =\left(\frac{\hat{C}_{\kappa, p, t}(u)}{u}\right)^{p} \operatorname{det}\left(1+\left\{\frac{u \hat{C}_{\kappa, p, t}^{\prime}(u)}{\hat{C}_{\kappa, p, t}(u)}-1\right\} \frac{\boldsymbol{v}^{\top} \boldsymbol{v}}{u^{2}}\right) \\
& =\hat{C}_{\kappa, p, t}^{\prime}(u)\left(\frac{\hat{C}_{\kappa, p, t}(u)}{u}\right)^{p-1},
\end{aligned}
$$

because of Sylvester's determinant identity, viz. $\operatorname{det}\left(I_{m \times m}+A_{m \times n} B_{n \times m}\right)=\operatorname{det}\left(I_{n \times n}+\right.$ $\left.B_{n \times m} A_{m \times n}\right)$. It follows from (23) and

$$
I_{\nu}^{\prime}(u)=I_{\nu-1}(u)-\frac{\nu}{u} I_{\nu}(u)
$$

see Abramowitz and Stegum (1972), 9.6.26, p. 376, that $A_{p}$ satisfies Riccati's differential equation

$$
A_{p}^{\prime}(u)+A_{p}^{2}(u)+\frac{p-1}{u} A_{p}(u)=1, \forall u>0 .
$$

Riccati's equation, (18), (19) and some algebraic manipulations lead to (20). Differentiating (18) w.r.t. $r$ yields

$$
\frac{\mathrm{d} \bar{u}}{\mathrm{~d} r}=\frac{1}{\hat{C}_{\kappa, p, t}^{\prime}(\bar{u})}=\left(\frac{r}{\bar{u}}\right)^{p-1} \hat{\sigma}_{\kappa, p, t}^{-2}(\bar{u})>0,
$$

which implies that the saddlepoint $\bar{u}$ is a continuous and increasing function of $r$.

II. The Legendre-Fenchel transform or convex conjugate of the convex function $f: \mathbb{R}^{p} \rightarrow \mathbb{R}$ is given by $\mathcal{L}(f)(\boldsymbol{y})=\sup _{\boldsymbol{v} \in D}\{\langle\boldsymbol{v}, \boldsymbol{y}\rangle-f(\boldsymbol{v})\}$, for $\boldsymbol{y} \in \mathbb{R}^{p}$ and where $D=\left\{\boldsymbol{v} \in \mathbb{R}^{p}|| f(\boldsymbol{v}) \mid<\right.$ $\infty\}$. From the multidimensional saddlepoint approximation, refer e.g. to Section 6.5 of Barndorff-Nielsen and Cox (1989), the saddlepoint approximation to $\hat{g}_{\boldsymbol{\mu}, \kappa, p, t}(\boldsymbol{y})$, the conditional density of $\hat{\boldsymbol{R}}_{t}$ given $\left\{N_{t}>0\right\}$ at $\boldsymbol{y} \in \mathbb{R}^{p}$, under the $\operatorname{vMF}(\boldsymbol{\mu}, \kappa)$ distribution and w.r.t. $l_{p}$, is given by

$$
\tilde{\hat{g}}_{\boldsymbol{\mu}, \kappa, p, t}(\boldsymbol{y})=(2 \pi)^{-\frac{p}{2}} \hat{\sigma}_{\kappa, p, t}^{-1}(\bar{u}) \exp \left\{-\mathcal{L}\left(\hat{K}_{\boldsymbol{\mu}, \kappa, t}\right)(\boldsymbol{y})\right\} .
$$

The Legendre-Fenchel transform appearing in this formula can be computed as follows,

$$
\begin{aligned}
\mathcal{L}\left(\hat{K}_{\boldsymbol{\mu}, \kappa, t}\right)(\boldsymbol{y}) & =\sup _{\boldsymbol{v} \in \mathbb{R}^{p}}\left\{\langle\boldsymbol{v}, \boldsymbol{y}\rangle-\hat{K}_{\boldsymbol{\mu}, \kappa, t}(\boldsymbol{v})\right\} \\
& =\sup _{\boldsymbol{v} \in \mathbb{R}^{p}}\left\{\langle\boldsymbol{v}-\kappa \boldsymbol{\mu}, \boldsymbol{y}\rangle-\hat{K}_{\boldsymbol{\mu}, \kappa, t}(\boldsymbol{v}-\kappa \boldsymbol{\mu})\right\} \\
& =\sup _{\boldsymbol{z} \in \mathbb{S}^{p-1}, u \geq 0}\left\{\langle u \boldsymbol{z}, r \boldsymbol{x}\rangle-\hat{K}_{\boldsymbol{\mu}, \kappa, t}(u \boldsymbol{z}-\kappa \boldsymbol{\mu})\right\}-\langle\kappa \boldsymbol{\mu}, \boldsymbol{y}\rangle \\
& =\sup _{u \geq 0}\left\{u r-\hat{\bar{K}}_{\kappa, t}(u)\right\}-\langle\kappa \boldsymbol{\mu}, \boldsymbol{y}\rangle \\
& =\mathcal{L}\left(\hat{\bar{K}}_{\kappa, t}\right)(r)-\langle\kappa \boldsymbol{\mu}, \boldsymbol{y}\rangle \\
& =\bar{u} r-\hat{\bar{K}}_{\kappa, t}(\bar{u})-\kappa r\langle\boldsymbol{\mu}, \boldsymbol{x}\rangle
\end{aligned}
$$


where $\bar{u}$ is uniquely defined by (18). The fourth of the above equalities is due to $\hat{K}_{\boldsymbol{\mu}, \kappa, t}(u \boldsymbol{z}-$ $\kappa \boldsymbol{\mu})=\hat{\bar{K}}_{\kappa, t}(u)$. The sixth and last equality follows from the strict convexity and the differentiability of $\hat{\bar{K}}_{\kappa, t}$. We can now join this last result with (27) and obtain the saddlepoint approximation at $\boldsymbol{y} \in \mathbb{R}^{p}$ as

$$
\tilde{\hat{g}}_{\boldsymbol{\mu}, \kappa, p, t}(\boldsymbol{y})=(2 \pi)^{-\frac{p}{2}} \hat{\sigma}_{\kappa, p, t}^{-1}(\bar{u}) \hat{\bar{M}}_{\kappa, t}(\bar{u}) \mathrm{e}^{-\bar{u} r} \exp \{\kappa r\langle\boldsymbol{\mu}, \boldsymbol{x}\rangle\}
$$

III. It follows from (2) and (3) that, under the $\operatorname{vMF}(\boldsymbol{\mu}, \kappa)$ distribution, the joint conditional Lebesgue density of the polar angles and the resultant length $\left(\hat{\alpha}_{1, t}, \ldots, \hat{\alpha}_{p-1, t}, \hat{R}_{t}\right)$ factorizes as

$$
\begin{aligned}
\hat{w}_{\boldsymbol{\mu}, \kappa, p, t}^{\circ}\left(\alpha_{1}, \ldots, \alpha_{p-1}, r\right)= & \hat{g}_{\boldsymbol{\mu , \kappa , p , t}}\left(\boldsymbol{g}\left(\alpha_{1}, \ldots, \alpha_{p-1}, r\right)\right) J\left(\alpha_{1}, \ldots, \alpha_{p-2}, r\right) \\
= & {\left[\frac{a_{p}}{f_{p}\left(\boldsymbol{g}\left(\alpha_{1}, \ldots, \alpha_{p-1}, 1\right) \mid \boldsymbol{\mu}, \kappa r\right)} \hat{g}_{\boldsymbol{\mu}, \kappa, p, t}\left(\boldsymbol{g}\left(\alpha_{1}, \ldots, \alpha_{p-1}, r\right)\right) r^{p-1}\right] } \\
& \cdot \frac{f_{p}\left(\boldsymbol{g}\left(\alpha_{1}, \ldots, \alpha_{p-1}, 1\right) \mid \boldsymbol{\mu}, \kappa r\right)}{a_{p}} \prod_{j=2}^{p-1} \sin ^{p-j} \alpha_{j-1},
\end{aligned}
$$

$\forall \alpha_{1}, \ldots, \alpha_{p-2} \in[0, \pi], \alpha_{p-1} \in[0,2 \pi)$ and $r>0$, where $\boldsymbol{g}$ is the diffeomorphism (1). It follows from this last formula and from (9) of the Second polar factorization lemma that the squared bracket of the above formula is the conditional density of $R_{t}$, that is,

$$
\hat{q}_{\kappa, p, t}(r)=\frac{a_{p}}{c_{p}(\kappa r) \mathrm{e}^{\kappa r\langle\boldsymbol{\mu}, \boldsymbol{y}\rangle}} \hat{g}_{\boldsymbol{\mu}, \kappa, p, t}(\boldsymbol{y}) r^{p-1}, \quad \forall r>0 .
$$

By inserting the saddlepoint approximation in the Euclidean space (28) into this last formula, we obtain the saddlepoint approximation

$$
\tilde{\hat{q}}_{\kappa, p, t}(r)=\frac{a_{p}}{c_{p}(\kappa r)}(2 \pi)^{-\frac{p}{2}} \hat{\sigma}_{\kappa, p, t}^{-1}(\bar{u}) \hat{\bar{M}}_{\kappa, t}(\bar{u}) r^{p-1} \mathrm{e}^{-\bar{u} r}, \quad \forall r>0
$$

viz. (17).

In agreement with (11), the saddlepoint approximation of Theorem 2.4 does not depend on $\boldsymbol{\mu}$.

Corollary 2.5 (Saddlepoint approximation to the survival function). Let $r$ and $t>0$, then the saddlepoint approximation to the survival function $\overline{\hat{Q}}_{\kappa, p, t}(r)=\mathrm{P}_{\boldsymbol{\mu}, \kappa}\left[\hat{R}_{t} \geq r \mid N_{t}>0\right]$ is given by

$$
\begin{aligned}
\tilde{\hat{\hat{Q}}}_{\kappa, p, t}(r)=\hat{b}_{\kappa, p, t} 2^{1-\frac{p}{2}} \Gamma^{-1}\left(\frac{p}{2}\right) & \int_{\bar{u}}^{u^{*}} u^{p-1} \hat{\sigma}_{\kappa, p, t}(u) M_{N_{t}^{0}} \circ \log \frac{c_{p}(\kappa)}{c_{p}(u)} \\
& \cdot c_{p}^{-1}\left(\kappa \hat{C}_{\kappa, p, t}(u)\right) \exp \left\{-u \hat{C}_{\kappa, p, t}(u)\right\} \mathrm{d} u,
\end{aligned}
$$

where the lower integration bound $\bar{u}$ is the saddlepoint at $r$, implicitly defined by (18), the upper integration bound is $u^{*}=\lim _{s \rightarrow \infty} \bar{u}(s), \bar{u}(s)$ denoting the saddlepoint $\bar{u}$ at $s>0$, and where $\hat{\sigma}_{\kappa, p, t}(u)$ is given by (20). The normalizing constant $\hat{b}_{\kappa, p, t}$ can be obtained with the above integral with $\bar{u}=0$. 
Proof. This approximation to the survival function is the integral w.r.t. $s$ of the saddlepoint approximation to the density $\tilde{\hat{q}}_{\kappa, p, t}(s)$, given by (17), with the change of variable from $s$ to $u$ defined by $\hat{C}_{\kappa, p, t}(u)=s$, i.e. by the saddlepoint equation (19). This exempts the numerical computation of the saddlepoints over the integration grid. The integrand is then simplified by using

$$
s^{p-1}(u) \frac{\mathrm{d} s(u)}{\mathrm{d} u}=\hat{C}_{\kappa, p, t}^{p-1}(u) \hat{C}_{\kappa, p, t}^{\prime}(u)=u^{p-1} \hat{\sigma}_{\kappa, p, t}^{2}(u)
$$

refer to $(20)$.

It is direct to verify that the saddlepoint approximations given in Theorem 2.4 and Corollary 2.5 simplify to the following results: to the approximations given in Proposition 3.4 of Gatto (2017a), when $p=2$; to the approximations given in Theorem 2.9 and Corollary 2.10 of Gatto $(2017 \mathrm{c})$, when $\kappa=0$; and to the approximations given in Theorem 3.6 and the following tail probability formula of Gatto (2017b), when $N_{t}=n$, for any fixed $n \in\{1,2, \ldots\}$.

An important application of Theorem 2.4 and Corollary 2.5 is when the counting component $\left\{N_{t}\right\}_{t \geq 0}$ is the inhomogeneous Poisson process, precisely the Poisson random measure with mean measure $\Lambda$ over $([0, \infty), \mathcal{B}([0, \infty)))$. In this case $\Lambda$ is absolutely continuous w.r.t. the Lebesgue measure $l_{1}$ and, $\forall 0 \leq s<t<\infty, \mathrm{E}\left[N_{t}-N_{s}\right]=\Lambda((s, t])$. With the simplified notation $\Lambda(t)=\Lambda((0, t])$, we have $p_{n}(t)=\mathrm{e}^{-\Lambda(t)} \Lambda^{n}(t) / n !, \forall n \in\{0,1, \ldots\}$. The required m.g.f. of the zero-truncated process at time $t, N_{t}^{0}$, is given by

$$
M_{N_{t}^{0}}(v)=\frac{1-\mathrm{e}^{\Lambda(t) \mathrm{e}^{v}}}{1-\mathrm{e}^{\Lambda(t)}}, \forall v \in \mathbb{R} .
$$

The required first two derivatives of the c.g.f. are

$$
K_{N_{t}^{0}}^{\prime}(v)=\frac{\Lambda(t) \mathrm{e}^{v}}{1-\mathrm{e}^{-\Lambda(t) \mathrm{e}^{v}}} \text { and } K_{N_{t}^{0}}^{\prime \prime}(v)=\frac{\Lambda(t) \mathrm{e}^{v}}{1-\mathrm{e}^{-\Lambda(t) \mathrm{e}^{v}}}\left(1-\frac{\Lambda(t) \mathrm{e}^{v}}{\mathrm{e}^{\Lambda(t) \mathrm{e}^{v}}-1}\right), \forall v \in \mathbb{R}
$$

In this case $\left\{\boldsymbol{R}_{t}\right\}_{t \geq 0}$ is the inhomogeneous compound Poisson process with von Mises-Fisher step directions.

\section{Numerical comparisons}

Consider the von Mises-Fisher c.t.r.w. with number of direction changes over time modulated by the homogeous Poisson process with rate $\lambda>0$. Thus $\Lambda(t)=\lambda t, \forall t \geq 0$.

We first consider the conditional density of the total distance $\hat{R}_{t}$ given $\left\{N_{t}>0\right\}$ of the c.t.r.w. with $p=3, \lambda=1, t=8$. The concentrations $\kappa=1 / 2$ and 3 are considered respectively in Figures 1 and 2. The value of $\boldsymbol{\mu}$ is irrelevant. In these figures, the continuous curve is the normalized saddlepoint approximation and the histogram is obtained from 50000 conditional simulations. We can see that the saddlepoint approximations are accurate, in particular in the right-tail, which is the most important for applications. In 
Figure 2 we note some irregularities on the left tail of the Monte Carlo distribution. In this situation the saddlepoint approximation cannot reproduce these irregularities but acts like as smoother. Figure 3 considers the conditional densities of the total distance $\hat{R}_{t}$ with $p=3, \lambda=1, t=8$ and $\kappa=2,4,6$, appearing respectively from the left to the right. It shows the evolution of the density w.r.t. the directional concentration $\kappa$. Figure 4 considers the conditional densities of the total distance $\hat{R}_{t}$ with $\lambda=1, t=8, \kappa=3$ and $p=3,5,7$, appearing respectively from the right to the left. It shows the evolution of the density w.r.t. the dimension $p$.

These computations are performed with Matlab. The related computer programs are available at http://www.stat.unibe.ch.

\section{References}

Abramowitz, M., Stegun, I. E. (1972), Handbook of Mathematical Functions with Formulas, Graphs, and Mathematical Tables, Dover. (9-th printing conform to the 10-th original printing.)

Barndorff-Nielsen, O. E., Cox, D. R. (1989), Asymptotic Techniques for Use in Statistics, Chapman \& Hall.

Daniels, H. E. (1954), "Saddlepoint approximations in statistics", The Annals of Mathematical Statistics, 25, 631-650.

Gatto, R. (2017a), "Large deviations approximations to distributions of the total distance of compound random walks with von Mises directions", Methodology and Computing in Applied Probability, 19, 843-864.

Gatto, R. (2017b), "Saddlepoint approximations to the distribution of the total distance of the multivariate isotropic and von Mises-Fisher random walks", Mathematical Methods of Statistics, 26, 20-36.

Gatto, R. (2017c), "Saddlepoint approximation to the distribution of the total distance of the continuous time random walk", The European Physical Journal B, Condensed Matter and Complex Systems, Topical issue: "Continuous Time Random Walk Still Trendy: Fiftyyear History, Current State and Outlook", editors Kutner and Masoliver, 90: 238.

Jensen, J. L. (1995), Saddlepoint Approximations, Oxford University Press.

Mardia, K. V., Jupp, P. E. (2000), Directional Statistics, Wiley.

Montroll E. W., Weiss G. H. (1965), "Random Walks on Lattices. II", Journal of Mathematical Physics, 6, 167.

Shmueli, U., Weiss, G. H. (1995), Introduction to Crystallographic Statistics, International Union of Crystallography Book Series, 6, Oxford University Press. 
Figure 1: Saddlepoint approximation and histogram of simulations with $\operatorname{vMF}(\boldsymbol{\mu}, 1 / 2)$ directions and Poisson number of steps with $p=3, \lambda=1$ and $t=8$

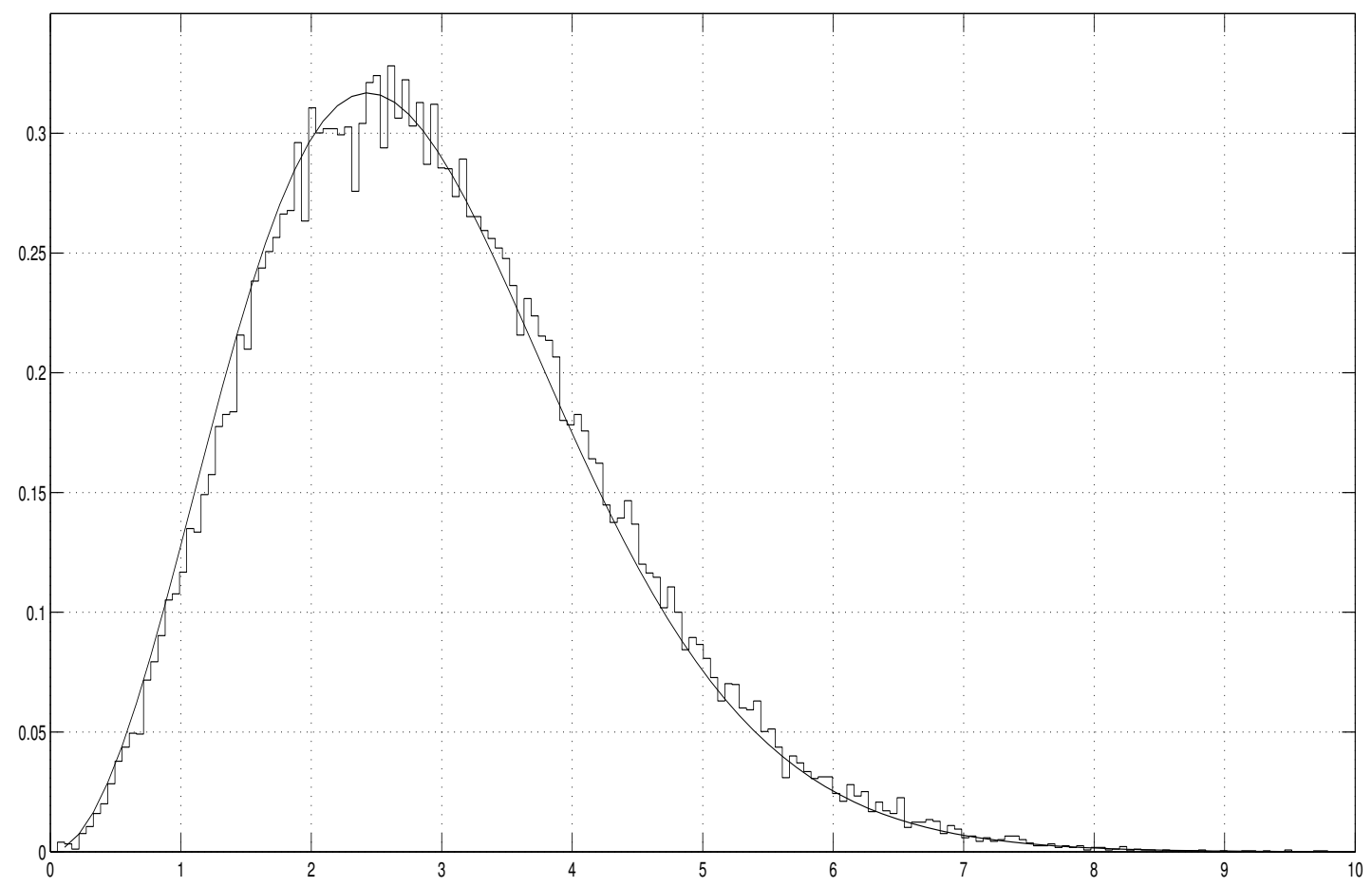


Figure 2: Saddlepoint approximation and histogram of simulations with $\operatorname{vMF}(\boldsymbol{\mu}, 3)$ directions and Poisson number of steps with $p=3, \lambda=1$ and $t=8$

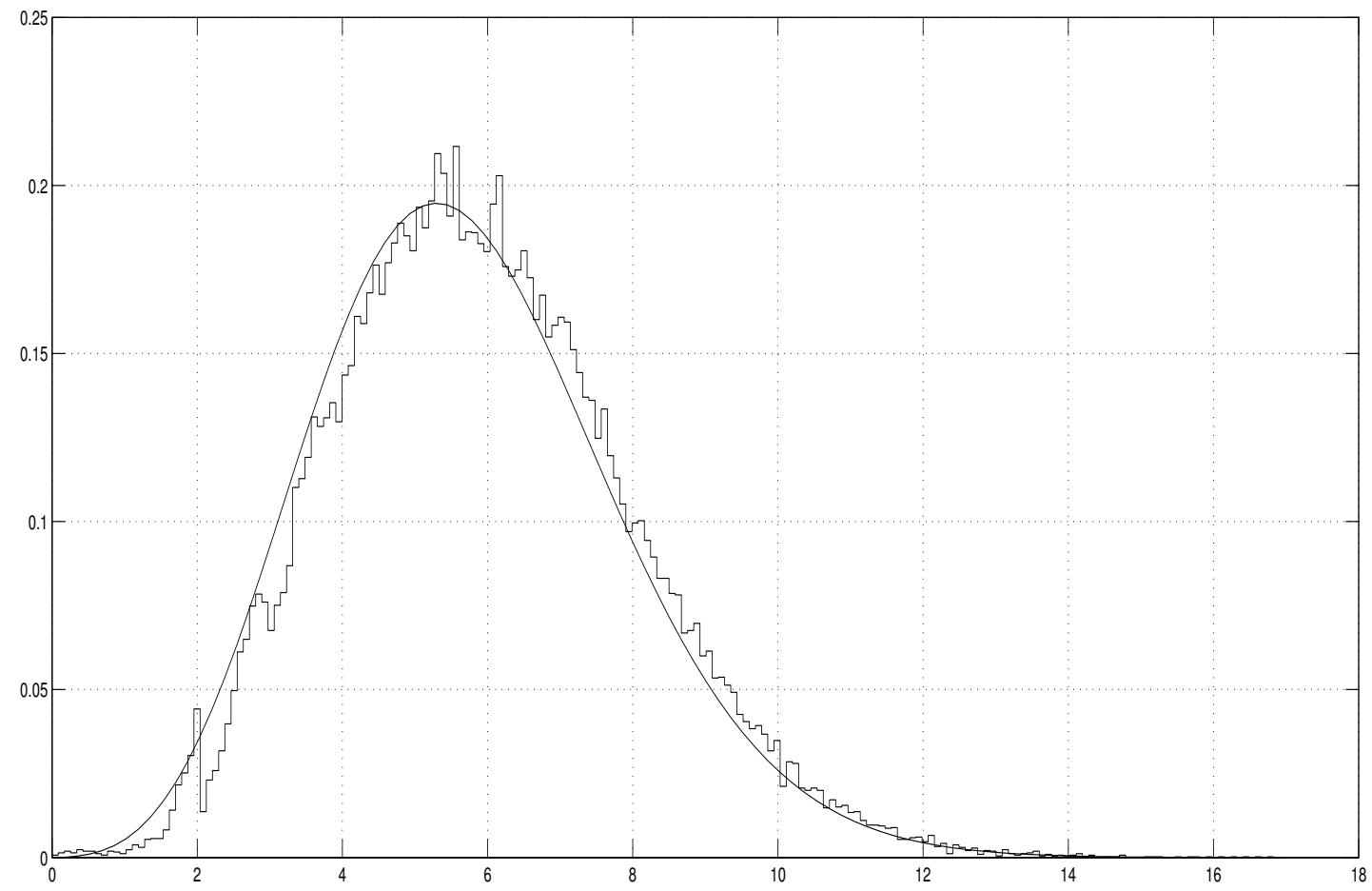


Figure 3: Saddlepoint approximations with $\operatorname{vMF}(\boldsymbol{\mu}, \kappa)$ directions and Poisson number of steps with $p=3, \lambda=1, t=8$ and $\kappa=2,4,6$, appearing respectively from left to right

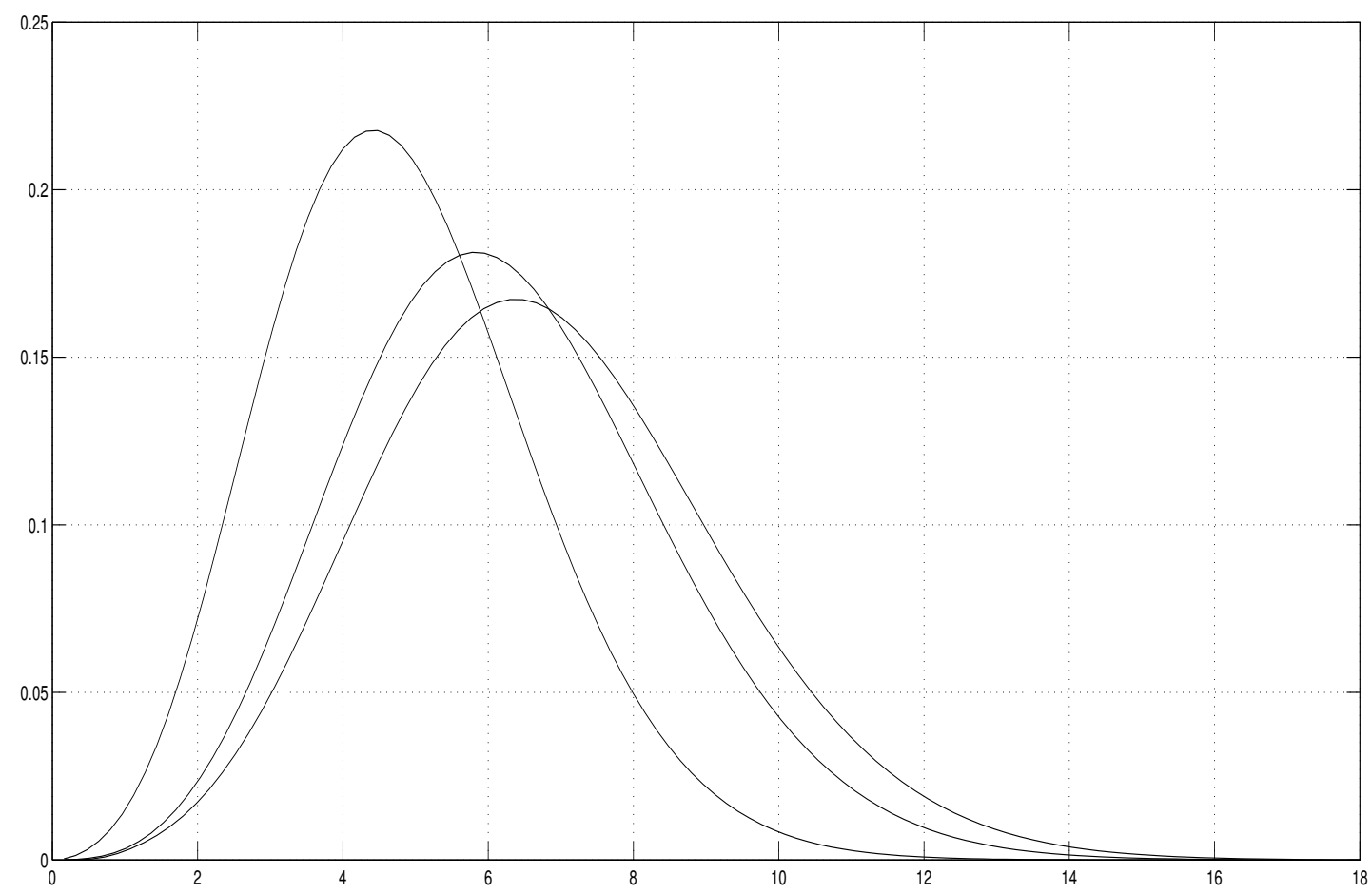


Figure 4: Saddlepoint approximations with $\operatorname{vMF}(\boldsymbol{\mu}, 3)$ directions and Poisson number of steps with $\lambda=1, t=8$ and $p=3,5,7$, appearing respectively from right to left

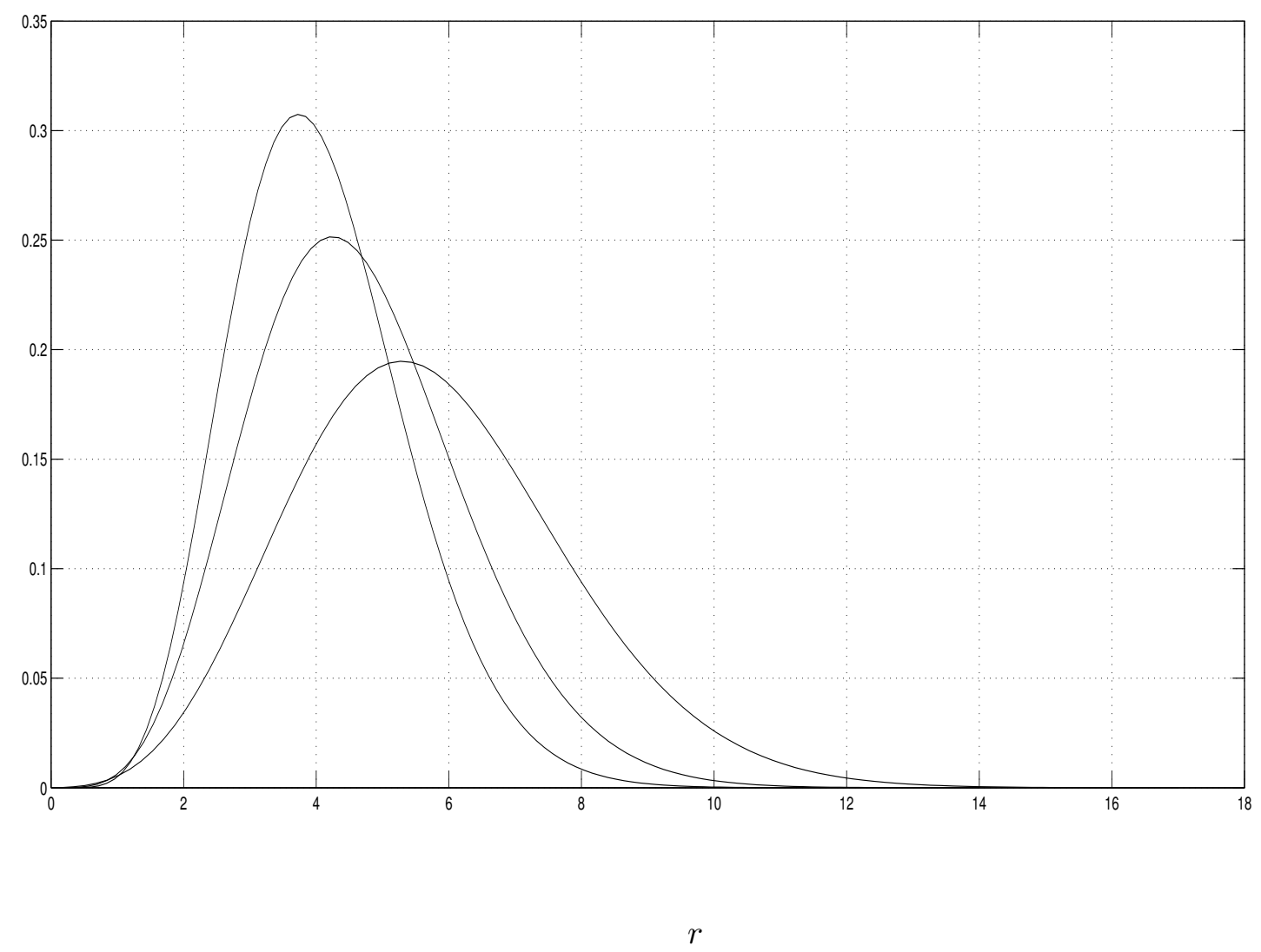

